\title{
Wozu Philosophie?
}

Erhard Taverna

Was ist eine «wohlwollende Lüge»?

Fakultät II für Kultur- und Sozialwissenschaften der Universität Luzern diskutiert. Als Erste sprach die Internistin Beatrice E. Kaufmann aus Arlesheim über ihre Arbeit «Die Lüge im Sprechzimmer - Gibt es Umstände, die es dem Arzt erlauben, zu seinen Patienten nicht ganz ehrlich zu sein?» Schweizerisches Zivilgesetzbuch und Bundesgericht relativieren die Pflicht des Arztes zur Aufklärung. Juristisch gilt das «therapeutische Privileg» nach dem Grundsatz, dem Patienten nicht zu schaden.

An einem konkreten Fallbeispiel wurden die medizinethischen Probleme nach utilitaristischen (Bentham) und nach deontologischen Kriterien (Kant) analysiert und dabei die eigene Haltung mitreflektiert. Das schriftliche Abfassen habe viel Formulierungsarbeit erfordert, aber auch Spass gemacht, die Denkweise verändert und die Interessen hinsichtlich Medizin und Recht erweitert. Was ist eine «wohlwollende Lüge», wird im Sprechzimmer immer gelogen/manipuliert? Die lebhaft Diskutierenden profitierten vom Erkenntnisgewinn der Hausärztin.

Als Zweiter sprach der Pädiater Hansjakob Roelli aus Sursee über seine Arbeit «Impfzwang im Pflegeberuf - Aspekte der Gerechtigkeit im Spannungsfeld von Patientenschutz und persönlicher Freiheit der Pflegenden». Das Spital kennt viele Akteure, Patienten, Ärzte, Pflegende, Führungsorgane und politische Behörden, alle mit unterschiedlichen Rollen, Interessen und Bedürfnissen, die oft sehr schwierig auf einen Nenner zu bringen sind. Die Auseinandersetzungen mit den Theorien der Gerechtigkeit (Rawls, Rauprich, Daniels) habe unter anderem auch gezeigt, wie persönliche Werthaltungen Theorie und Praxis beeinflussen. Auch ihm habe die Philosophie im eigenen Denken neue Wege geöffnet und das eigene Tun aus einer erkenntnistheoretischen Perspektive überprüfen lassen. Das bewusste Wahrnehmen verschiede- ner «Weltversionen» habe letztlich den Umgang mit Eltern und Patienten offener, anspruchsvoller und bereichernder gemacht.

Andreas Brenner, Professor an der Universität Basel, moderierte die Diskussionen und referierte anschliessend zum Thema «Den Menschen erkennen, zwischen Maschine und Leib». Ein kurzer Abriss zur Philosophiegeschichte vom Skeptiker Descartes über Julien Offray de la Mettrie mit seinem berühmten Werk «L'homme Machine» ebnete den Weg zu Husserl, Merleau Ponty, Plessner und vor allem zu Hermann Schmitz, dessen Neue Phänomenologie heute in der Philosophie und Medizin angekommen ist. Ein grosser Teil der psychosomatisch genannten Krankheiten hat, gemäss Schmitz, seinen Sitz weder im Körper noch in der Seele, sondern im Leib, von wo sie auf den Körper ausstrahlen. Jeder spürt Schmerz, Hunger, Durst, Schreck, Wollust, Behagen, Frische, Mattigkeit,

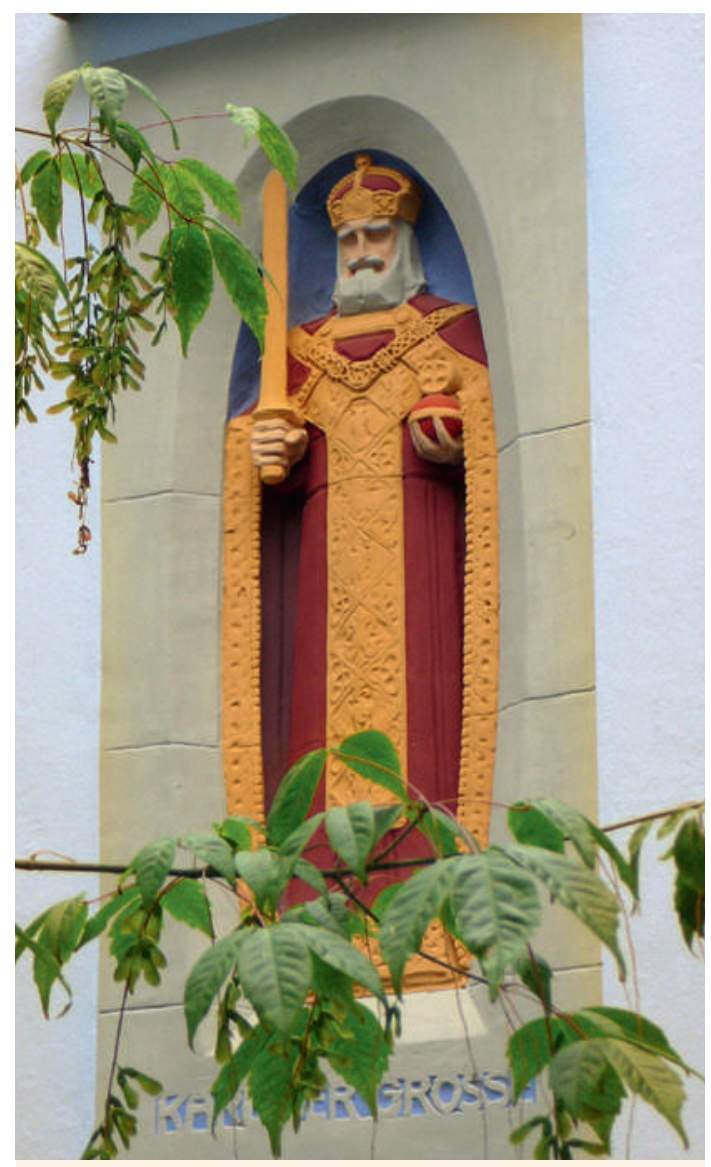

War offen für neue Ideen und Gelehrsamkeit: Karl der Grosse, nach dem ein Veranstaltungszentrum in Zürich benannt wurde. Beim Forum Medizin \& Philosophie gab es eine Vielzahl neuer Gedanken. 


\section{Forum Medizin \& Philosophie}

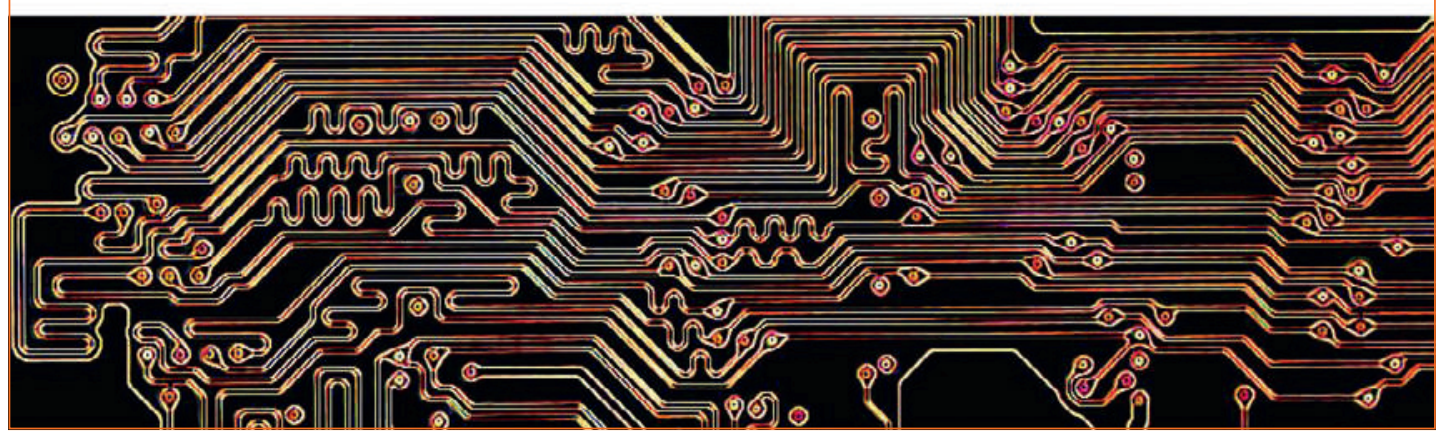

Weitere Informationen zum Forum Medizin \& Philosophie und zum Anlass am 25. Oktober auf http://fomep.ch.

Ein- und Ausatmen als Beispiele leiblicher Regungen, die weder sicht- noch tastbar sind. Schmitz widerlegt die herkömmliche Meinung, die den Menschen in Seele und Körper zerlegt. Nach ihm gibt es ein eigenständiges Gegenstandsgebiet des Spürens am eigenen Leib. Der 1928 in Leipzig geborene Philosoph hat aus seiner fundierten Kritik dualistischer Denkweisen ein eigenes Leibverständnis von «Regungsherden mit eigentümlicher Struktur und Dynamik» entwickelt, der Psychologie haben diagnostische und therapeutische Methoden nach Erkenntnissen dieser Gefühlsphilosophie entwickelt, die heute auch Fachrichtungen der Traumatologie übernehmen. Einige Positionen der Neuen Phänomenologie erinnern an Ideen der deutschen Romantik, etwa wenn Schmitz Gefühle zu Atmosphären ausweitet, die als geschichtsmächtige, übersubjektive Mächte unser Schicksal bestimmen.

\section{Ein Deutungssystem, dem in Zeiten dominierender, digitaler Maschinen- technik eine ausgleichende Funktion zukommen könnte.}

eine umfassende Phänomenologie des Empfindens und Wahrnehmens. Ein Hauptgedanke seiner Theorie ist die Unterscheidung zwischen dem Gefühl selbst und dem affektiven Betroffensein davon, für das Schmitz, mangels Alltagssprache, ein umfassendes und gut verständliches Begriffs-Alphabet geschaffen hat. Pflegende Berufe, aber auch gewisse Richtungen
Dank dem Forum Medizin \& Philosophie - eine instruktive Einführung in ein innovatives und kreatives Deutungssystem, dem in Zeiten dominierender, digitaler Maschinentechnik, vor allem im medizinischen Denken und Handeln eine ausgleichende Funktion zukommen könnte.

\section{Forum Medizin \& Philosophie - der nächste Anlass}

Teilnehmerinnen und Teilnehmer des Zertifikatskurses (CAS) «Philosophie und Medizin» der Universität Luzern und weitere philosophisch interessierte Ärzte haben am 28. Juni 2012 das «Forum Medizin und Philosophie» gegründet. Die Mitglieder des Forums treffen sich regelmässig in Arbeitsgruppen zu kritischen Reflexionen über die grundlegenden Denkformen, die in der Medizin als Wissenschaft und als Praxis wirksam sind, und suchen nach konstruktiven Vorschlägen, wie sich die Medizin in einem fortlaufenden Prozess weiterentwickeln kann und soll. Regelmässig finden Vereinsanlässe mit Beteiligung von Philosophen statt, die mit ihrer alternativen Sichtweise die Diskussion bereichern und Raum für neue kreative Lösungen schaffen.

Der nächste Anlass findet statt am Samstag, 25. Oktober 2014, im Hotel Uto Kulm auf dem Üetliberg. Auf dem Programm stehen unter anderem Vorträge des Philosophen Dr. Andreas Cremonini zum Thema "Leib und Sinn» und des Satirikers, Kolumnisten und Psychoanalytikers PD Dr. Peter Schneider zum Thema «Wie sozial sind psychische Krankheiten?» mit anschliessender Diskussion durch die TeilnehmerInnen. Der Anlass steht auch für Nichtmitglieder des Forums offen. 